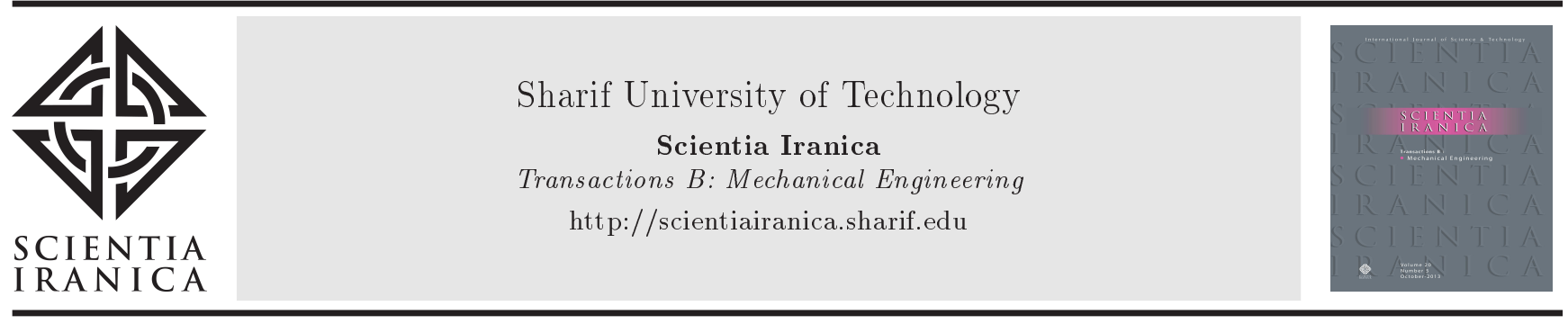

\title{
Stability of thixotropic fluids in pipe flow
}

\author{
M.H. Nahavandian, M. Pourjafar, and K. Sadeghy* \\ Center of Excellence in Design and Optimization of Energy Systems (CEDOES), School of Mechanical Engineering, College of \\ Engineering, University of Tehran, Tehran, P.O. Box 11155-4563, Iran.
}

Received 19 October 2016; received in revised form 26 December 2016; accepted 8 April 2017

\section{KEYWORDS}

Linear stability;

Thixotropic fluid;

Moore model;

Spectral method;

Pipe flow.

\begin{abstract}
Linear stability of a thixotropic fluid obeying the Moore model is investigated in pipe flow using a temporal stability analysis in which infinitesimally small perturbations, represented by normal modes, are superimposed on the base flow and their evolution in time is monitored in order to detect the onset of instability. An eigenvalue problem is obtained, which is solved numerically using the pseudo-spectral Chebyshev-based collocation method. The neutral instability curve is plotted as a function of the thixotropy number of the Moore model. Based on the results obtained in this work, it is concluded that the thixotropic behavior of the Moore fluid has a destabilizing effect on pipe flow.
\end{abstract}

(C) 2018 Sharif University of Technology. All rights reserved.

\section{Introduction}

Thixotropy appears to be a common effect among industrial fluids such as drilling muds [1-3]. Waxy crude oils are also known to exhibit thixotropic behavior at sufficiently low temperatures. The main cause of thixotropy in such complex fluid systems arises from an interaction between the fluid elements and the suspended particles or microstructures. In practice, these particles temporarily cross-link and form bonds, which are broken down by the action of shear. Simultaneously, these bonds are reformed through the Brownian motion, but, because structure reform takes place at a lower rate, in practice, one might witness a time-dependent thixotropic effect. For instance, the viscosity of such fluid systems may vary considerably with time in addition to its being a function of the shear rate [1-3]. Such time-dependent behavior is expected to affect the critical Reynolds number in confined flows $[4,5]$. In spite of its technological importance, instability of thixotropic fluids has rarely

*. Corresponding author. Tel/Fax: +982182084011

E-mail address: sadeghy@ut.ac.ir (K. Sadeghy)

doi: $10.24200 /$ sci. 2017.4498 been addressed in the past. One can notably mention the work carried out by Pearson and Tardy [6], and Ebrahimi et al. [7], who have shown that thixotropy has a stabilizing effect on the Saffman-Taylor instability in a Hele-Shaw cell. On the other hand, Pourjafar et al. [8] have shown that in circular Coeutte flow, the effect of thixotropy can be stabilizing or destabilizing depending on the gap size and the severity of the fluid's thixotropy. To the best of our knowledge, the effect of thixotropy on the stability of pipe flow has not been addressed in the past.

The interest in the stability of pipe flow stems from the fact that this particular geometry is widely used for the transport of Newtonian and nonNewtonian fluids [9-15]. In previous studies dealing with non-Newtonian fluids, the effect of shear-thinning and yield stress has already been investigated on the critical Reynolds number in pipe flow. For example, we already know that shear-thinning has a stabilizing effect on pipe flow [16]. Also, based on current knowledge, it is well established that a non-zero yield stress increases the critical Reynolds number in pipe flow $[17,18]$. As to thixotropic fluids, it is also known that thixotropy can have a destabilizing effect on plane Poiseuille flow [19]. Surprisingly, however, the effect of thixotropy on the critical Reynolds number in circular 
Poiseuille flow still remains unexplored. In the present work, we intend to investigate the effect of thixotropy on the critical Reynolds number in pipe flow. Our interest in this particular flow arises from the fact that pipelines are widely used for the transport of waxy crude oils, which are known to exhibit thixotropic effects. Another motivation for the present study is the fact that capillary rheometers are frequently used for the rheological characterization of non-Newtonian fluids including thixotropic ones [20]. As a matter of fact, the viscometric data obtained using capillary rheometers are commonly used for deciding on their constitutive equation. With these in mind, this paper addresses the stability to small disturbances of circular Poiseuille flow for a typical thixotropic fluid. To that end, the paper is organized as follows. In the next section, the equations of motion are presented for the thixotropic fluid of interest (namely, the Moore model). The basic (i.e., unperturbed) solution is then described for this particular fluid. After that, we proceed with developing the linear stability analysis of the basic flow so-obtained. The numerical solution to the stability problem is then given together with presenting our understanding of its significance. The work is concluded by highlighting major findings.

\section{Mathematical formulation}

Figure 1 shows the flow geometry, which is a horizontal circular rigid pipe of length $L$ and radius $R$ with its axis along the $z$-direction. The pipe is assumed to be carrying an incompressible thixotropic fluid under laminar conditions. Neglecting gravity effects and assuming axial symmetry, the equations governing the isothermal flow of any fluid are the Cauchy equations of motion together with the continuity equation. In cylindrical co-ordinate systems, they are written as:

$$
\begin{aligned}
& \rho\left(\frac{\partial u_{r}}{\partial t}\right.\left.+u_{r} \frac{\partial u_{r}}{\partial r}+u_{z} \frac{\partial u_{r}}{\partial z}-\frac{u_{\theta}^{2}}{r}\right)=-\frac{\partial p}{\partial r} \\
&+\frac{1}{r} \frac{\partial\left(r \tau_{r r}\right)}{\partial r}+\frac{\partial \tau_{z r}}{\partial z}-\frac{\tau_{\theta \theta}}{r} \\
& \rho\left(\frac{\partial u_{\theta}}{\partial t}+\right.\left.u_{r} \frac{\partial u_{\theta}}{\partial r}+u_{z} \frac{\partial u_{\theta}}{\partial z}-\frac{u_{\theta} u_{r}}{r}\right)=\frac{1}{r^{2}} \frac{\partial\left(r^{2} \tau_{r \theta}\right)}{\partial r} \\
&+\frac{\partial \tau_{z r}}{\partial z}, \\
& \rho\left(\frac{\partial u_{z}}{\partial t}+u_{r} \frac{\partial u_{z}}{\partial r}+u_{z} \frac{\partial u_{z}}{\partial z}\right)=-\frac{\partial p}{\partial z}+\frac{1}{r} \frac{\partial\left(r \tau_{r z}\right)}{\partial r} \\
&+\frac{\partial \tau_{z z}}{\partial z}, \\
& \frac{1}{r} \frac{\partial}{\partial r}\left(r u_{r}\right)+\frac{\partial u_{z}}{\partial z}=0
\end{aligned}
$$

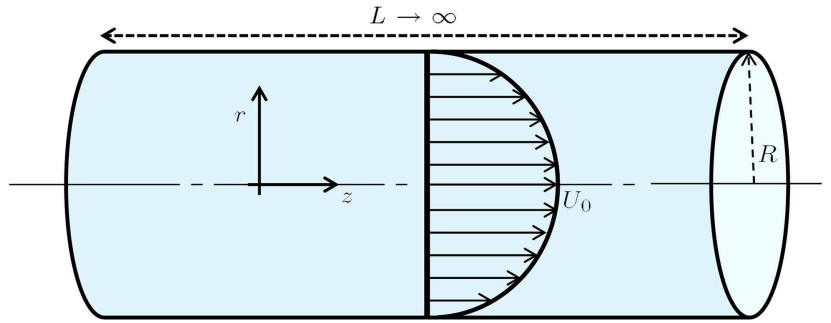

Figure 1. Schematics of the circular pipe flow.

where $\rho$ is the fluid's density; $u_{r}, u_{\theta}$, and $u_{z}$ are the velocity components; and $\tau_{i j}$ are the components of the stress tensor.

The stress components in these equations need a constitutive equation to be related to the velocity field. In this study, we assume that the thixotropic fluid of interest obeys the "Moore model" [21,22]. For a fluid obeying this simple thixotropic model, the viscosity is both shear- and time-dependent, that is:

$$
\mu(\dot{\gamma}, t)=\mu_{\infty}(1+\alpha \lambda(\dot{\gamma}, t))
$$

where, $\dot{\gamma}$ is an effective shear rate defined by the relationship:

$$
\dot{\gamma}=\sqrt{2 d_{i j} d_{i j}}
$$

where $d_{i j}$ is the rate-of-deformation tensor defined by:

$$
\begin{aligned}
& D=\mathrm{d}_{\mathrm{ij}}= \\
& {\left[\begin{array}{ccc}
\frac{\partial u_{r}}{\partial r} & \frac{1}{2}\left[\frac{1}{r}\left(\frac{\partial u_{r}}{\partial \theta}-u_{\theta}\right)+\frac{\partial u_{\theta}}{\partial r}\right] & \frac{1}{2}\left(\frac{\partial u_{r}}{\partial z}+\frac{\partial u_{z}}{\partial r}\right) \\
d_{21}=d_{12} & \frac{1}{r}\left(\frac{\partial u_{\theta}}{\partial \theta}+u_{r}\right) & \frac{1}{2}\left(\frac{\partial u_{\theta}}{\partial z}+\frac{1}{r} \frac{\partial u_{z}}{\partial \theta}\right) \\
d_{31}=d_{13} & d_{32}=d_{23} & \frac{\partial u_{z}}{\partial z}
\end{array}\right](5 \mathrm{c})}
\end{aligned}
$$

In Eq. (5), $\lambda(\dot{\gamma}, t)$ is the structural parameter, which varies between 1 to 0 with "one" denoting complete structure buildup and "zero" denoting complete structure breakdown. The viscosities corresponding to these two limiting cases are referred to by the zero-shear $\left(\mu_{0}\right)$ and infinite-shear $\left(\mu_{\infty}\right)$ viscosities, respectively. It is worth mentioning that in the Moore model, these limiting viscosities are related to each other through $\alpha$, the simple relationship: $\alpha=\frac{\mu_{0}-\mu_{\infty}}{\mu_{\infty}}$. To represent the time-dependency of the structural parameter, an appropriate kinetic equation is needed. In the Moore model, the kinetic equation takes the following form $[21,22]$ :

$$
\frac{D \lambda}{D t}=-k_{1} \dot{\gamma} \lambda+k_{2}(1-\lambda)
$$

where $k_{1}$ and $k_{2}$ are model parameters controlling the rates of structure breakdown and structure buildup, respectively. While $k_{2}$ has the dimension of a reciprocal time, $k_{1}$ is dimensionless. Thus, the ratio $K=k_{1} / k_{2}$ can be regarded as the "characteristic 
time" of the Moore fluid. In a sense, $K$ controls the time needed by the Moore to find its new configuration under equilibrium conditions. Still, it should be noted that for thixotropic effects to become important, $K$ should be of the same order or even larger than the "characteristic time of the flow".

\subsection{The basic flow}

As the first step in our instability analysis, we have to find out the velocity and viscosity fields under steady conditions-the so-called basic flow. Since the flow is unidirectional, the equations of motion are simply reduced to:

$$
0=-\frac{\partial p}{\partial z}+\frac{1}{r} \frac{\partial}{\partial r}\left(r \tau_{r z}\right)
$$

where $p$ is the isotropic pressure, $r$ is the radial distance from the pipe axis, $z$ is the distance along the pipe, and $\tau_{r z}=G r / 2$ is the shear stress (with $G$ being the driving pressure gradient $\partial p / \partial z)$. On the other hand, under equilibrium conditions, the structural parameter is obtained as $\lambda_{s s}=k_{2} /\left(k_{1} \dot{\gamma}_{s s}+k_{2}\right)$ so that the equilibrium shear stress becomes:

$$
\tau_{s s}(r)=\mu_{\infty}\left(1+\alpha \frac{k_{2}}{k_{1} \dot{\gamma}_{s s}+k_{2}}\right) \dot{\gamma}_{s s}
$$

where the subscript "ss" means steady state. Having multiplied this equation by $k_{2} / k_{1} \mu_{\infty}$, after some simple rearrangement, we end up with the following quadratic equation for the steady shear rate:

$$
\dot{\gamma}_{s s}^{2}+\left((1+\alpha) \frac{k_{2}}{k_{1}}-\frac{\tau_{s s}}{\mu_{\infty}}\right) \dot{\gamma}_{s s}-\frac{k_{2}}{k_{1} \mu_{\infty}} \tau_{s s}=0 .
$$

In an attempt to solve this equation for the shear rate, we first try to make it dimensionless. To that end, we scale the radial position by $R$, the shear rate by $U_{0} / R$, and the shear stress by $G R$, where $U_{0}$ is the velocity scale (say, the centerline velocity). From Eq. (9), the physically acceptable root is easily obtained as:

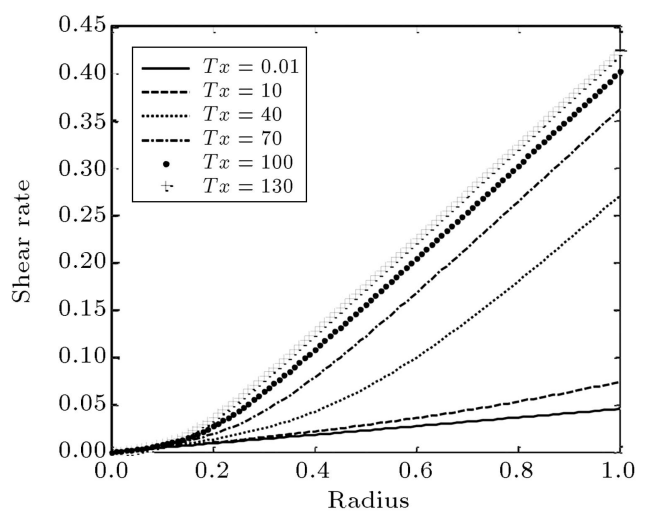

(a)

$$
\dot{\gamma}_{s s}=\frac{1}{2}\left[\frac{r}{2}-\frac{(1+\alpha)}{T x}+\sqrt{\left(\frac{r}{2}-\frac{(1+\alpha)}{T x}\right)^{2}+\frac{2 r}{T x}}\right],
$$

where $T x=k_{1} G R / \mu_{\infty} k_{2}$ is called the thixotropy number. Figure 2 shows a plot of Eq. (10) as a function of the thixotropic parameter, $T x$, and the viscosity ratio, $\alpha$.

Having found the shear rate profiles at equilibrium, we can obtain the basic velocity profiles by simply integrating the equation: $\dot{\gamma}_{s}=d u_{z} / d r$. In dimensionless form, the result is:

$$
\begin{aligned}
u_{z}(r)= & \frac{\left(1-r^{2}\right)}{8}+\frac{r-1}{2}\left(\frac{1+\alpha}{T x}\right)-\frac{1}{2}\left(\frac{r}{2}+\frac{1-\alpha}{T x}\right) \\
& \times \sqrt{\frac{r^{2}}{4}+r\left(\frac{1-\alpha}{T x}\right)+\left(\frac{1+\alpha}{T x}\right)^{2}} \\
& +2\left[\operatorname { l n } \left(\frac{2-2 \alpha}{T x}+r\right.\right. \\
& \left.\left.+2 \sqrt{\frac{r^{2}}{4}+r\left(\frac{1-\alpha}{T x}\right)+\left(\frac{1+\alpha}{T x}\right)^{2}}\right)\right] \\
& \left.+\frac{-\alpha}{T x^{2}}\right]-2\left[\operatorname { l n } \left(\frac{2-2 \alpha}{T x}+1\right.\right. \\
& \left.\left.+2 \sqrt{\frac{1}{4}+\frac{1-\alpha}{T x}+\left(\frac{1+\alpha}{T x}\right)^{2}}\right)\right] \cdot\left[\frac{-\alpha}{T x^{2}}\right] \\
& +\frac{1}{2}\left(\frac{1}{2}+\frac{1-\alpha}{T x}\right) \\
& \sqrt{\frac{1}{4}+\frac{1-\alpha}{T x}+\left(\frac{1+\alpha}{T x}\right)^{2}} \cdot
\end{aligned}
$$

As expected, this equation reduces to the well-known parabolic velocity profile for Newtonian fluids by sim-

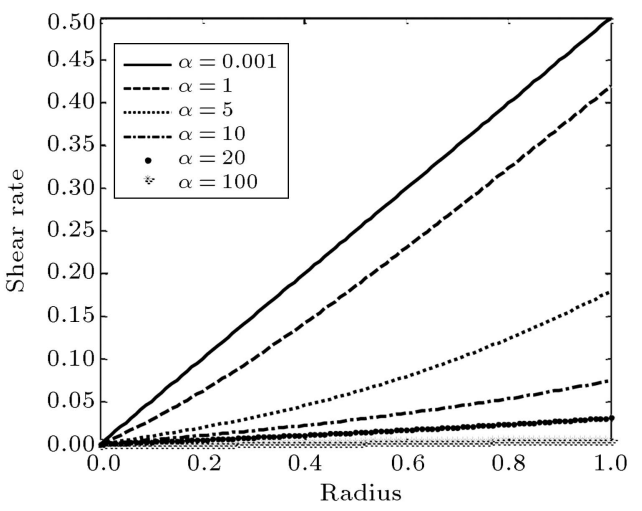

(b)

Figure 2. Shear rate profiles as a function of the thixotropic number, $T x$, and the viscosity ratio, $\alpha$. 
ply setting $\alpha=0$. The importance of this semianalytical solution is that it can easily be used to investigate the effect of $T x$ and $\alpha$ on the velocity profiles. As such, it can be regarded as an exact solution for the pipe flow of Moore fluids. And, as we know, exact solutions are quite rare in nonNewtonian fluid dynamics-mainly because of the nonlinearity of their constitutive behavior. This is perhaps why the field of non-Newtonian fluid mechanics relies so much on the computational techniques. However, computer codes need to be verified first and this should preferably be done by comparing their output with an exact solution. To this should be added the fact that exact solutions can provide us with a better insight as to the physics of any given fluid mechanics problem. Obviously, exact solutions are indispensable tools in the field of fluid mechanics, and this by itself highlights the importance of Eq. (11) by itself. It is worth mentioning that our semi-analytical solution has enabled us to find the velocity profiles without any need to add diffusion terms to the kinetic equation as previously used by Billingham and Ferguson [23]-see also [19] who resorted to the same trick in order to obtain converged results in their fluid mechanics problem.

Figures 3 and 4 show typical basic-flow velocity profiles using Eq. (11). As can be seen in Figure 3, by an increase in $\alpha$, the velocity profiles deviate more and more from the parabolic profile of Newtonian fluids. This is because at sufficiently large $\alpha$, the logarithmic term in Eq. (11) becomes progressively more important. It is also interesting to note that the centerline velocity decreases by an increase in the viscosity ratio. Figure 4 shows the effect of $T x$ number on the axial velocity profile for a given $\alpha$. As can be seen in this figure, the centerline velocity increases by an increase in $T x$. Obviously, the two parameters in the Moore model (i.e., $\alpha$ and $T x$ ) have opposite effects on the velocity profiles.

\section{Linear stability analysis}

Having found the basic solution, we can now proceed with their linear stability analysis. Using the idea of normal modes, we substitute:

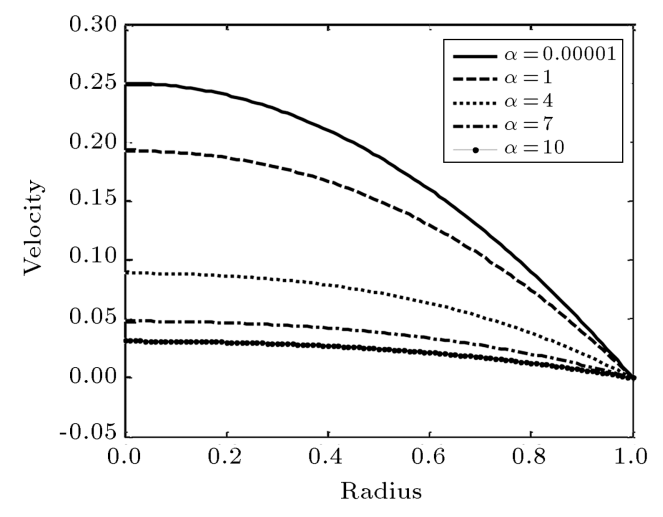

(a)

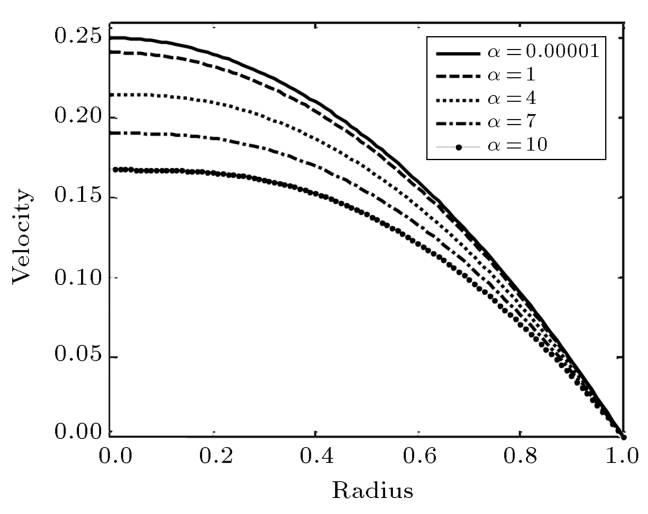

(b)

Figure 3. Effect of the viscosity ratio on the axial basic flow velocity profiles, $u$, obtained at (a) $T x=10$ and (b) $T x=100$.

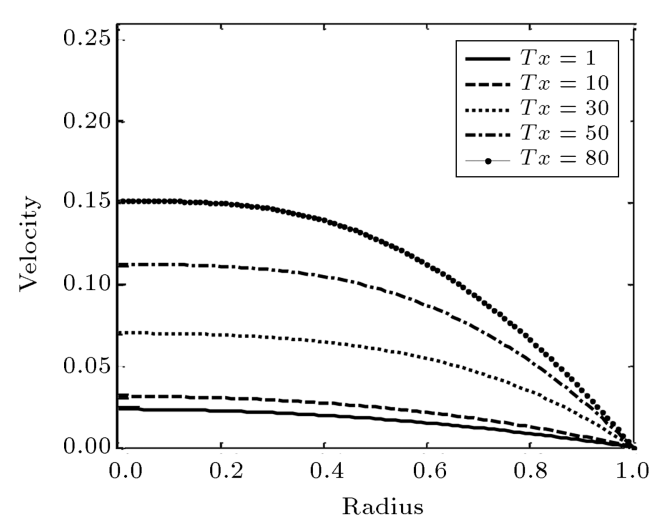

(a)

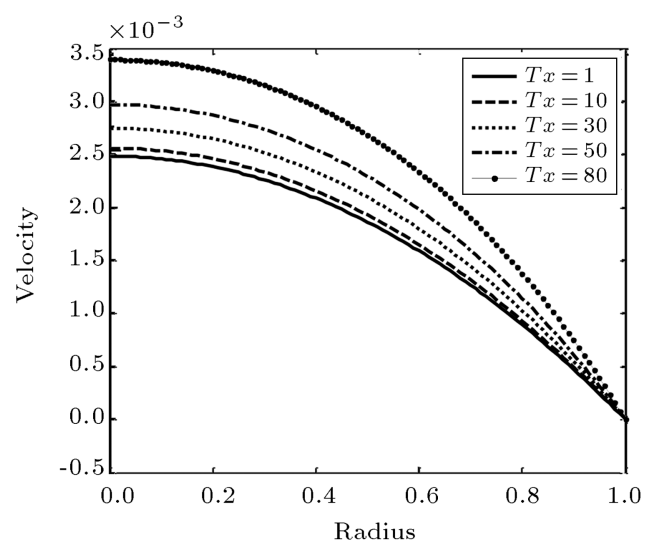

(b)

Figure 4. Effect of the viscosity ratio on the axial basic flow velocity profiles $u$ in various Tx numbers: (a) $\alpha=10$ and (b) $\alpha=100$. 


$$
\begin{aligned}
& u_{z}(r, z, t)=\bar{u}_{z}+\hat{u}_{z}=\bar{u}_{z}+\hat{w}(r) e^{q t+i k z} \\
& u_{\theta}(r, z, t)=0+\hat{u}_{\theta}=\hat{f}(r) e^{q t+i k z} \\
& u_{r}(r, z, t)=0+\hat{u}_{r}=\hat{u}(r) e^{q t+i k z} \\
& \lambda(r, z, t)=\bar{\lambda}+\hat{\varepsilon}(r) e^{q t+i k z} \\
& \nu(r, z, t)=\bar{\nu}+\hat{y}(r) e^{q t+i k z} \\
& p(r, z, t)=\bar{p}+\hat{\pi}(r) e^{q t+i k z}
\end{aligned}
$$

where $\bar{u}_{z}, \bar{p}, \bar{\lambda}$, and $\bar{\nu}$ represent the basic solution as represented by Eqs. (10) and (11), and $\nu$ is the kinematic viscosity. It needs to be mentioned that, based on the temporal instability analysis, in these equations, the axial wavenumber $(k)$ is a real number whereas the growth rate $(q)$ is a complex number. Now, as the next step, we insert these equations into the time-dependent set of the governing equations. After neglecting the nonlinear terms based on the "linear stability analysis," we end up with the following equations for the perturbations amplitude functions:

$$
\begin{aligned}
& \hat{u} q+i k \bar{u}_{z} \hat{u}=\frac{-1}{\rho} \frac{d \hat{\pi}}{d r}+\frac{2}{r} \bar{\nu} \frac{d \hat{u}}{d r}+2 \frac{d \bar{\nu}}{d r} \frac{d \hat{u}}{d r}+2 \bar{\nu} \frac{d^{2} \hat{u}}{d r^{2}} \\
& +i k \hat{y} \frac{d \bar{u}_{z}}{d r}-k^{2} \bar{\nu} \hat{u} \\
& \hat{f} q+i k \hat{f} \bar{u}_{z}=\frac{-\bar{\nu} \hat{f}}{r^{2}}-\frac{\hat{f}}{r} \frac{d \bar{\nu}}{d r}+\frac{\bar{\nu}}{r} \frac{d \hat{f}}{d r}+\frac{d \bar{\nu}}{d r} \frac{d \hat{f}}{d r} \\
& +\bar{\nu}^{2} \frac{d^{2} \hat{f}}{d r^{2}}-k^{2} \bar{\nu} \hat{f} \\
& q \hat{w}+\hat{f} \frac{d \bar{u}_{z}}{d r}+i k \hat{w} \bar{u}_{z}=\frac{-i k \hat{\pi}}{\rho} \\
& \quad+\frac{1}{r}\left\{\begin{array}{l}
\frac{d \bar{\nu}}{d r} \frac{d \hat{w}}{d r}+\bar{\nu} \frac{d^{2} \hat{w}}{d r^{2}}+\frac{d \hat{y}}{d r} \frac{d \bar{u}_{z}}{d r}+ \\
\hat{y} \frac{d^{2} \bar{u}_{z}}{d r^{2}}+i k \hat{u} \frac{d \bar{\nu}}{d r}+i k \bar{\nu} \frac{d \hat{u}}{d r}
\end{array}\right\}-2 k^{2} \hat{w} \bar{\nu} \\
& \frac{\hat{u}}{r}+\frac{d \hat{u}}{d r}+i k \hat{w}=0 .
\end{aligned}
$$

To calculate the viscosity function in these equations, the effective shear rate should be calculated first from
Eq. (5a). To that end, we first write down the rate of deformation tensor as shown in Box I (see Eq. (5b)), where we have dropped all $\partial / \partial \theta$ terms thanks to the flow being axisymmetric in cylindrical coordinate system. The effective shear rate can then be obtained as:

$$
\left[\begin{array}{l}
\quad\left[\left(\frac{\partial \hat{u}_{r}}{\partial r}\right)^{2}+\left(\frac{\hat{u}_{r}}{r}\right)^{2}+\left(\frac{\partial\left(\bar{u}_{z}+\hat{u}_{z}\right)}{\partial z}\right)^{2}\right\} \\
+\left(\frac{\partial \hat{u}_{\theta}}{\partial z}\right)^{2}+\left(\frac{\partial \hat{u}_{r}}{\partial z}\right)^{2}+\left(\frac{\partial\left(\bar{u}_{z}+\hat{u}_{z}\right)}{\partial r}\right)^{2} \\
+2 \frac{\partial \hat{u}_{r}}{\partial z} \frac{\partial\left(\bar{u}_{z}+\hat{u}_{z}\right)}{\partial r}+\frac{\hat{u}_{\theta}^{2}}{r^{2}}+\left(\frac{\partial \hat{u}_{\theta}}{\partial r}\right)^{2} \\
-2 \frac{\partial \hat{u}_{\theta}}{\partial r} \frac{\hat{u}_{\theta}}{r} .
\end{array}\right.
$$

This equation can be simplified as:

$$
\dot{\gamma} \approx \sqrt{\left(\frac{\partial \bar{u}_{z}}{\partial r}\right)^{2}+2 \frac{\partial \hat{u}_{z}}{\partial r} \frac{\partial \bar{u}_{z}}{\partial r}+2 \frac{\partial \hat{u}_{r}}{\partial z} \frac{\partial \bar{u}_{z}}{\partial r}} .
$$

The above equation can be recast into the following form:

$$
\begin{aligned}
\dot{\gamma} & =\sqrt{\left(\frac{\partial \bar{u}_{z}}{\partial r}\right)^{2}\left[1+2 \frac{\partial \hat{u}_{z / \partial r}}{\partial \bar{u}_{z / \partial r}}+2 \frac{\partial \hat{u}_{r / \partial z}}{\partial \bar{u}_{z / \partial r}}\right]} \\
& =\left|\frac{\partial \bar{u}_{z}}{\partial r}\right| \sqrt{1+2\left(\frac{\partial \hat{u}_{z / \partial r}}{\partial \bar{u}_{z / \partial r}}+\frac{\partial \hat{u}_{r} / \partial z}{\partial \bar{u}_{z / \partial r}}\right)} .
\end{aligned}
$$

Having dropped all nonlinear terms and using the binomial, we obtain:

$$
\dot{\gamma} \approx S\left[1+\left(\partial \hat{u}_{z} / \partial r+\partial \hat{u}_{r} / \partial z\right)\right]
$$

where, " $S$ " refers to the sign of $\frac{d \bar{u}_{z}}{d r}$. Knowing the shear rate for the perturbed form of the Moore model, the kinetic equation (see Eq. (6)) takes the following form:

$$
\begin{aligned}
q \hat{\varepsilon} & +\hat{u} \frac{d \bar{\lambda}}{d r}+i k \bar{u}_{z} \hat{\varepsilon}=-k_{1} S\left(\bar{\lambda}\left(\frac{d \hat{w}}{d r}+i k \hat{u}\right)+\hat{\varepsilon}\right) \\
& -k_{2} \hat{\varepsilon} .
\end{aligned}
$$

$$
\mathbf{D}=\left[\begin{array}{ccc}
\frac{\partial \hat{u}_{r}}{\partial r} & \frac{1}{2}\left[\frac{1}{r}\left(\frac{\partial \hat{u}_{r}}{\partial \theta}-\hat{u}_{\theta}\right)+\frac{\partial \hat{u}_{\theta}}{\partial r}\right] & \frac{1}{2}\left(\frac{\partial \hat{u}_{r}}{\partial z}+\frac{\partial\left(\hat{u}_{z}+\bar{u}_{z}\right)}{\partial r}\right) \\
d_{21}=d_{12} & \frac{1}{r}\left(\frac{\partial \hat{u}_{\theta}}{\partial \theta}+\hat{u}_{r}\right) & \frac{1}{2}\left(\frac{\partial \hat{u}_{\theta}}{\partial z}+\frac{1}{r} \frac{\partial\left(\hat{u}_{z}+\bar{u}_{z}\right)}{\partial \theta}\right) \\
d_{31}=d_{13} & d_{32}=d_{23} & \frac{\partial\left(\hat{u}_{z}+\bar{u}_{z}\right)}{\partial z}
\end{array}\right] .
$$


It should also be noted that there is a relationship between the disturbed amplitude of the kinematic viscosity and the structural parameter itself. To obtain this relationship, Eq. (5) is re-written as:

$$
\begin{aligned}
\nu & =\nu_{\infty}[1+\alpha(\bar{\lambda}+\hat{\lambda})]=\nu_{\infty}(1+\alpha \bar{\lambda})+\alpha \nu_{\infty} \hat{\lambda} \\
& =\bar{\nu}+\alpha \nu_{\infty} \hat{\lambda} .
\end{aligned}
$$

On the other hand, knowing that $\nu=\bar{\nu}+\hat{\nu}$, we obtain:

$$
\hat{\varepsilon}=\left(1 / \alpha \nu_{\infty}\right) \hat{y} \text {. }
$$

Hence, Eq. (14e) can be re-written as:

$$
\begin{aligned}
& q \hat{y}+\hat{u} \frac{d \bar{\nu}}{d r}+i k \bar{u}_{z} \hat{y}=-k_{1} S\left(\left(\bar{\nu}-\nu_{\infty}\right)\left(\frac{d \hat{w}}{d r}+i k \hat{u}\right)\right. \\
& \quad+\hat{y})-k_{2} \hat{y} .
\end{aligned}
$$

As the next step, we substitute: $a=R k, \sigma=R^{2} q / \nu_{\infty}$, $u^{*}=\hat{u} / U_{0}, f^{*}=\hat{f} / U_{0}, \nu^{*}=\bar{\nu} / \nu_{\infty}$, and $y^{*}=\hat{y} / \nu_{\infty}$. We then combine Eqs. (13a) to (13c) with Eq. (14e) in order to obtain the following $r$ - and $\theta$-momentum equations. We finally end up with following equations for the dimensionless (still unknown) functions " $u$ " and " $f *$ ":

$$
\begin{aligned}
D^{2} \bar{\nu}^{*} & {\left[\frac{D^{2} u^{*}}{a^{2} r^{* 2}}+\frac{D u^{*}}{a^{2} r^{* 2}}-\frac{u^{*}}{R^{2} a^{2} r^{* 4}}+u^{*}\right] } \\
& +D \bar{\nu}^{*}\left[\left(\frac{1}{a^{2} r^{* 2}}-\frac{R^{2}}{a^{2}}\right) D^{3} u^{*}\right. \\
& +2\left(\frac{1}{a^{2} r^{2}}-\frac{1}{R a^{2} r^{3}}\right) D^{2} u^{*} \\
& +\left(\frac{1}{R^{2} a^{2} r^{4}}-\frac{2}{R a^{2} r^{3}}-\frac{1}{R r}\right) D u^{*} \\
& \left.-\left(\frac{2}{R r}+\frac{4}{R^{3} a^{2} r^{5}}-\frac{2}{R r^{3} a^{2}}\right) u^{*}\right] \\
& +\bar{\nu}^{*}\left[-\frac{R^{2} D^{4} u^{*}}{a^{2}}+\frac{D^{3} u^{*}}{a^{2} r^{* 2}}-D^{2} u^{*}\left(3-\frac{1}{R r^{*}}\right)\right. \\
& +i\left[\frac{u^{*} U_{0} a \bar{u}_{z}^{*}}{\nu_{\infty} R}-\frac{y^{*} R D^{3} \bar{u}_{z}^{*}}{a}-\frac{2 R D y^{*} D^{2} \bar{u}_{z}^{*}}{a}\right. \\
& -D u^{*}\left(-\frac{4}{R r^{*}}+\frac{1}{R^{2} r^{* 2}}\right) \\
& \left.-u\left(\frac{D^{2} u^{*}}{a^{2} r^{*} a^{2}}-\frac{2}{R^{2} r^{* 2}}-\frac{a^{2}}{R^{2}}\right)\right]
\end{aligned}
$$

$$
\begin{aligned}
& -\frac{1}{a} D \bar{u}_{z}^{*} R D^{2} y^{*}-\frac{1}{a} D \bar{u}_{z}^{*} R y^{*} \\
& \left.+\frac{a}{\nu_{\infty} R} U_{0}^{2} u^{*} \bar{u}_{z}^{*}\right] \\
& +\frac{i \operatorname{Re}}{a}\left(D \bar{u}_{z}^{*} D f^{*}-\bar{u}_{z}^{*} D^{2} u^{*}-D u^{*} D \bar{u}_{z}^{*}\right. \\
& \left.+f^{*} D^{2} \bar{u}_{z}^{*}-D \bar{u}_{z}^{*} D u^{*}\right)=0 .
\end{aligned}
$$

For the $\theta$-momentum equation, we obtain:

$$
\begin{aligned}
D \bar{\nu}^{*} & \left(-\frac{f^{*}}{R r^{*}}+D f^{*}\right)+\bar{\nu}^{*}\left(-\frac{f^{*} a^{2}}{R^{2}}-\frac{f^{*}}{R^{2} r^{* 2}}\right. \\
& \left.+\frac{1}{R r^{*}} D f^{*}\right)+\bar{\nu}^{* 2} \nu_{\infty} D^{2} f^{*}-i \frac{a \operatorname{Re}}{R^{2}} \bar{u}_{z}^{*} f^{*} \\
& -f^{*} \frac{\sigma}{R^{2}}=0
\end{aligned}
$$

where $D=\frac{d}{d r}$. As to the $z$-momentum equation, we have:

$$
\begin{aligned}
& y^{*}\left.\frac{\nu_{\infty} \sigma}{R^{2}}+i \frac{a}{R} \bar{u}_{z}^{*}+T x k_{2} S+k_{2} G R\right) \\
&=-2 i U_{0} T x k_{2} S\left(\begin{array}{c}
\frac{R}{a}\left(D^{2} u^{*}-\frac{u^{*}}{R^{2} r^{* 2}}+\frac{1}{R r^{*}} D u^{*}\right) \\
+\frac{a}{R} u^{*} U_{0}
\end{array}\right) \\
&\left(\bar{\nu}^{*}+1\right),
\end{aligned}
$$

where $y^{*}$ is defined as:

$$
y^{*}=\frac{\xi\left(\nu^{*}+1\right)}{\frac{\nu_{\infty} \sigma}{R^{2}}+i \frac{a}{R} \bar{u}_{z}+T x k_{2}{ }^{*} S+k_{2}{ }^{*} G R} .
$$

Also, $\xi$ is defined as:

$$
\begin{aligned}
\xi= & -2 i U_{0} T x k_{2}{ }^{*} S\left(\frac{R}{a}\left(D^{2} u^{*}-\frac{u^{*}}{R^{2} r^{* 2}}+\frac{1}{R r^{*}} D u^{*}\right)\right. \\
& \left.+\frac{a}{R} u^{*} U_{0}\right) .
\end{aligned}
$$

The above set of differential equations (governing the perturbations) needs six boundary conditions to be amenable to a numerical solution. Based on the no-slip condition, the three velocity perturbations and their amplitudes must vanish at the wall. On the other hand, from Eq. (13d) the boundary condition on $w$ can be translated into $\frac{d \hat{u}}{d r}+\frac{\hat{u}}{r}=0$ at the wall as well as at the centerline. To capture the first mode of instability, the dimensionless amplification factor, $\sigma$, should be set equal to zero. 


\section{Method of solution}

Since our main target is to find out the critical Reynolds number, it suffices to obtain the neutral stability curves by finding the critical wave number at which we have: $\max \left[\operatorname{real}\left(\sigma_{c r}\left(\operatorname{Re}_{c r}, \alpha_{c r}\right)\right)\right]=0$. In the MATLAB code, we used the "polyig" function to deal with the eigenvalue problem resulting from Eqs. (15a) and (15b). In addition, for solving the stability equations, we have relied on the pseudo-spectral based on Chebyshev polynomials and using the Gauss-Lobatto collocation points [24]. The fundamental consideration of spectral method is to assume unknown functions of $u^{*}$ and $f^{*}$ as the base functions, that is:

$$
u^{*}(x)=\sum_{n=0}^{N} a_{n} T_{n}(x), \quad f^{*}(x)=\sum_{n=0}^{N} b_{n} T_{n}(x),
$$

where $T_{n}(x)=\cos \left(n \cos ^{-1}(x)\right)$ is the $n$th Chebyshev polynomials. Since the polynomials are defined in $[-1,1]$, we use the transformation $x=2 r-1$ to map the interval of $[0,1]$ (from pipe centerline to its wall) to $[-1,1]$. The collocation points used in this work are the Gauss-Lobatto points defined by:

$$
x_{j}=-\cos \left(j \frac{\pi}{N}\right), \quad j=0,1,2, \ldots, N .
$$

In the next section, we report instability results obtained in this way for the critical Reynolds number as a function of $\alpha$ and $K$ (or, $T x$ number). We have checked the effect of the number of base (or, trial) functions on the accuracy of our numerical results and reached the conclusion that using $N=60$ can ensure grid-independent results. To validate the code, we can rely on published Newtonian results for pipe flow of Newtonian fluids. In [25], the critical Reynolds number for Newtonian fluids has been reported to be equal to 2000. Our spectral code renders a value of roughly 2004, which is quite close to that reported in this reference (see Figure 5).

\section{Results and discussions}

Having verified the code, we are now ready to present our new results addressing the effect of a fluid's thixotropy, as represented by the thixotropy number $(T x)$, on the instability picture in pipe flow. For completeness, we also address the effect of viscosity ratio on the critical Reynolds number. Figure 6 shows the effect of the thixotropy ratio on the critical Reynolds number as well as on the critical wave number for several viscosity ratios, $\alpha$. As can be seen in this figure, for any given $\alpha$, by an increase in $T x$, the critical Reynolds number is decreased monotonically, meaning that the thixotropy ratio in the Moore model has a destabilizing effect on the pipe flow. Interestingly, longer wave numbers (or,

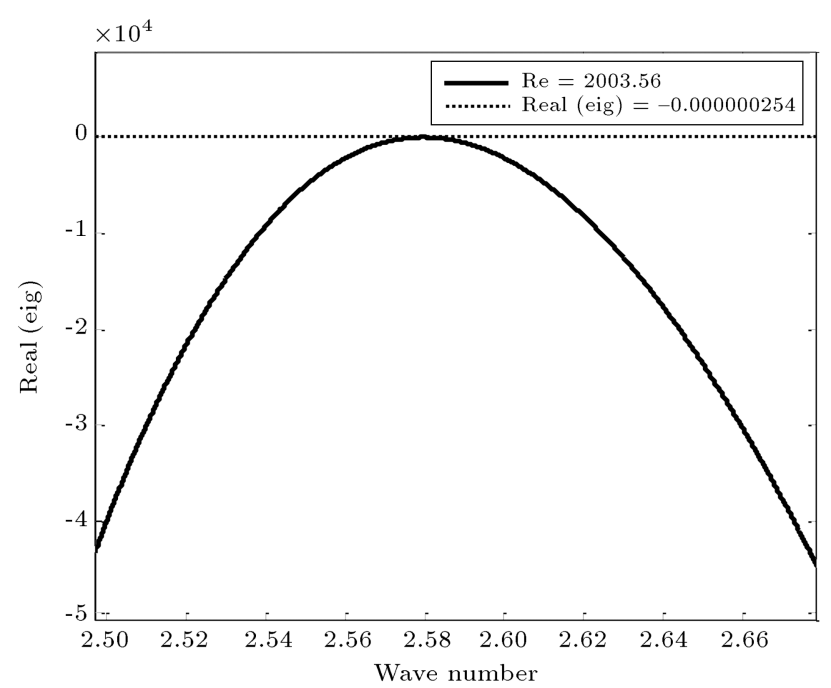

Figure 5. Critical Reynolds number for Newtonian fluids.

equivalently, shorter wavelengths) are excited when $T x$ is increased (see Figure 6(b), right). Unfortunately, a fluid obeying Moore model is shear-thinning in addition to being thixotropic. One might therefore wonder if the effect shown in Figure 6 is a true reflection of the thixotropy or not. Based on available data (see, for example, Ref. [16]), we already know that shear-thinning has a stabilizing effect on pipe flow. Therefore, the results presented in Figure 6 show that the destabilizing effect of thixotropy is so strong that it has eclipsed the stabilizing effect of shear-thinning, so much so that the net effect is destabilizing (see Figure 6).

Figure 7 shows the effect of the viscosity ratio on the critical Reynolds number as well as on the critical wave number for several thixotropy ratios, $T x$. As can be seen in this figure, for any given $T x$, by an increase in $\alpha$, the critical Reynolds number is increased monotonically, meaning that the viscosity ratio in the Moore model has a stabilizing effect on the pipe flow. Interestingly, shorter wave numbers (or, equivalently, longer wavelengths) are excited when $\alpha$ is increased (see Figure 7(b)). As mentioned above, a fluid obeying Moore model is shear-thinning and, for a given $\mu_{\infty}$, its shear-thinning behavior is intensified when $\alpha$ is increased. On the other hand, the effect of shearthinning on pipe flow is already known to be stabilizing. Thus, the destabilizing effect of the viscosity ratio is not surprising (see Figure 7).

The strong effects of the thixotropy number and the viscosity ratio in the Moore model on the instability picture of pipe flow can best be seen by plotting the neutral instability curves in Figure 8. In Figure 8(a), we have shown the effect of $T x$ for a typical $\alpha=10$ and in the right plot, we have shown the effect of $\alpha$ for a typical $T x=10$. The destabilizing effect of $T x$ is evident in the left plot (cf. results at $T x=10$ with results at $T x=100$ ). Similarly, the stabilizing effect of 


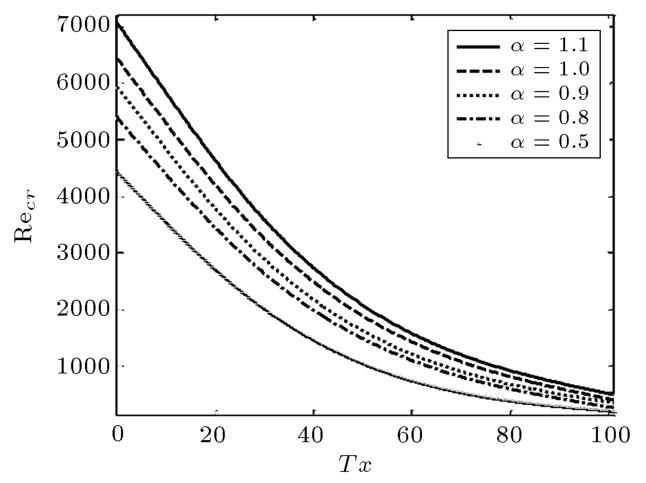

(a)

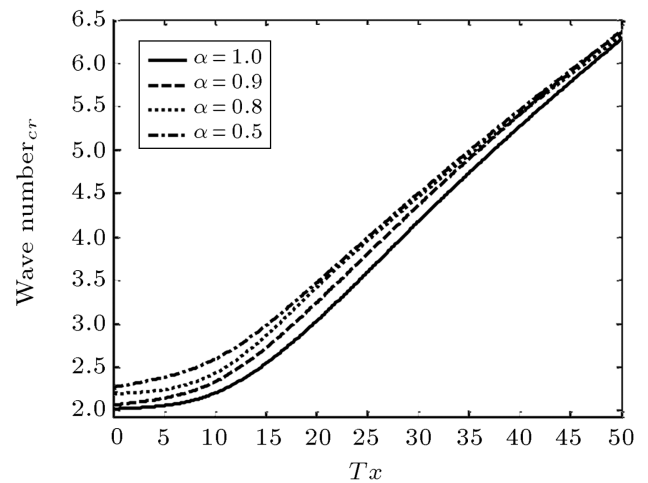

(b)

Figure 6. Effect of the thixotropy number, $T x$, on the critical Reynolds number.

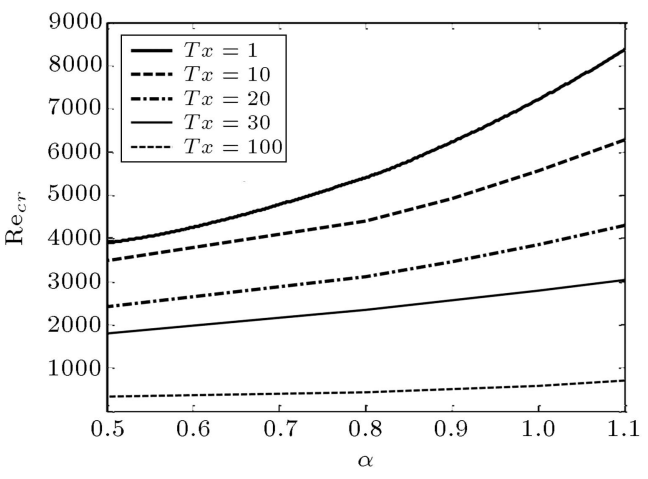

(a)

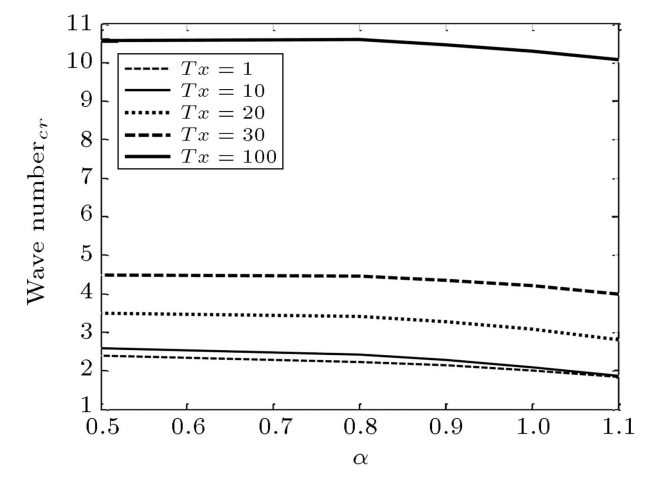

(b)

Figure 7. Effect of the viscosity ratio, $\alpha$, on the critical Reynolds number.

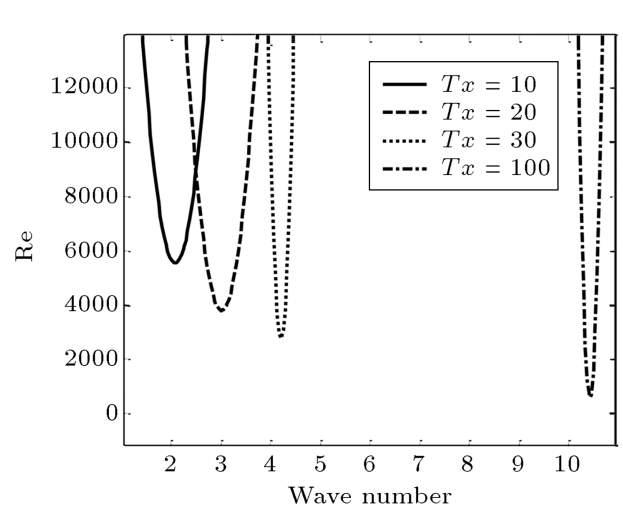

(a)

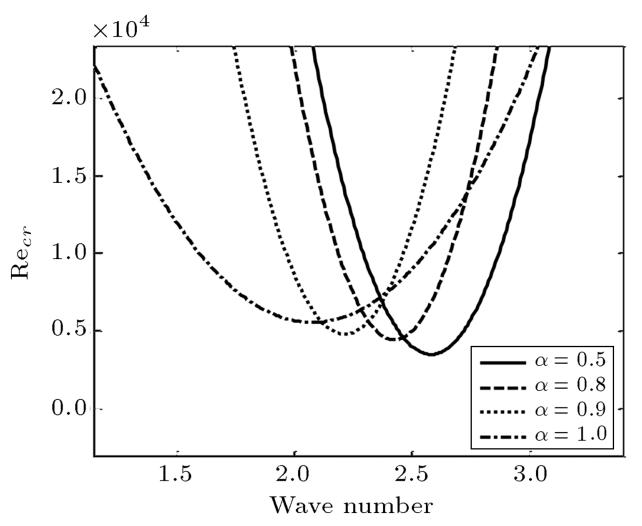

(b)

Figure 8. Effect of the thixotropy ratio at constant viscosity ratio: (a) $\alpha=10$ and viscosity ratio at constant $T x$ number and (b) $T x=10$ on the critical Reynolds number.

$\alpha$ is clear in the right plot (cf. results at $\alpha=0.5$ with results at $\alpha=1$ ).

\section{Concluding remarks}

In the present work, we have relied on the Moore model to investigate the effect of a fluid's thixotropy on the critical Reynolds number in pipe flow using a linear, temporal, normal-mode, and stability analysis. Based on the results obtained in the present work, it can be concluded that thixotropic fluids obeying Moore model are less stable than their corresponding Newtonian fluids. That is to say that, the thixotropy number (i.e., the breakdown-to-rebuild ratio) has a destabilizing effect on the pipe flow. Since a fluid obeying Moore model is both shear-thinning and thixotropic, interpretation of the results appear to be a tricky task at first sight. However, Fortunately, because shearthinning is already known to have a stabilizing effect on pipe flow, the destabilizing effect found for the Moore 
model can be ascribed to its thixotropic behavior, which is so strong that it eclipses the stabilizing effect of shear-thinning.

\section{References}

1. Mewis, J. "Thixotropy-a general review", Int. J. of Non-Newt. Fluid Mech., 6, pp. 1-20 (1979).

2. Barnes, H.A. "Thixotropy-a review", Int. J. of NonNewt. Fluid Mech., 70(1-2), pp. 1-33 (1997).

3. Mewis, J. and Wagner, N.J. "Thixotropy", Adv. Colloid and Interface Sci, 147/148, pp. 214-227 (2009).

4. Chandrasekhar, S., Hydrodynamic and Hydromagnetic Stability, Oxford University Press, London (1961).

5. Drazin, P.G. and Reid, W.H., Hydrodynamic Stability, 2nd edition. Camb. Univ. Press, Cambridge (2004).

6. Pearson, J.R.A. and Tardy, P.M.J. "Models for flow of non-Newtonian and complex fluids through porous media", Int. J. of Non-Newt. Fluid Mech., 102, pp. 447-473 (2002).

7. Ebrahimi, B., Taghavi, S.M., and Sadeghy, K. "Twophase viscous fingering of immiscible thixotropic fluids: A numerical study", Int. J. of Non-Newt. Fluid Mech., 218, pp. 40-52 (2015).

8. Pourjafar, M., Chaparian, E., and Sadeghy, K. "Taylor-Couette instability of thixotropic fluids", Meccanica, 50, pp. 1451-1465 (2015).

9. Wygnanski, I. and Champagne, F. "On transition in a pipe. Part 1. The origin of puffs and slugs and the flow in a turbulent slug", J. of Fluid Mechanics, 59, pp. 281-335 (1973).

10. Leite, R.J. "An experimental investigation of the stability of Poiseuille flow", J. of Fluid Mechanics, 5, pp. 81-96 (1959).

11. Eliahou, S., Tumin, A., and Wygnanski, I. "Laminarturbulent transition in Poiseuille pipe flow subjected to periodic perturbation emanating from the wall", $J$. of Fluid Mechanics, 361, pp. 333-349 (1998).

12. Darbyshire, A. and Mullin, T. "Transition to turbulence in constant-mass-flux pipe flow", J. of Fluid Mechanics, 289, pp. 83-114 (1995).

13. Garg, V. and Rouleau, W. "Linear spatial stability of pipe Poiseuille flow", J. of Fluid Mechanics, 54, pp. 113-127 (1972).

14. Stuart, J. "Instability and transition in pipes and channels", Transition and Turbulence, pp. 77-94 (1981).

15. Zikanov, O.Y. "On the instability of pipe poiseuille flow", Physics of Fluids (1994-present), 8, pp. 29232932 (1996).

16. Güzel, B., Frigaard, I., and Martinez, D. "Predicting laminar-turbulent transition in Poiseuille pipe flow for non-Newtonian fluids", Chemical Engineering Science, 64, pp. 254-264 (2009).
17. Nouar, C. and Frigaard, I. "Nonlinear stability of Poiseuille flow of a Bingham fluid: theoretical results and comparison with phenomenological criteria", Int. J. of Non-Newt. Fluid Mech., 100, pp. 127-149 (2001).

18. Frigaard, I., Howison, S., and Sobey, I. "On the stability of Poiseuille flow of a Bingham fluid", J. of Fluid Mechanics, 263, pp. 133-150 (1994).

19. Frigaard, I. "On the stability of shear flows of suspensions", 7th International Congress on Rheology, August 3-8, Monterey, California (2008).

20. Macosko, C.W., Rheology: Principles, Measurements and Applications, 1st edition, Wiley VCH (1994).

21. Moore, F. "The rheology of ceramic slips and bodies", Trans. Br. Ceram. Soc., 58, p. 470 (1959).

22. Cheng, D.C.H. and Evans, F. "Phenomenological characterization of the rheological behavior of inelastic reversible thixotropic and antithixotropic fluids", $B r$. J. Appl. Phys., 16(11), pp. 1599-1617 (1965).

23. Billingham, J. and Ferguson, J.W.J. "Laminar, unidirectional flow of a thixotropic fluid in a circular pipe", Int. J. of Non-Newt. Fluid Mech., 47, pp. 21-55 (1993).

24. Nahavandian, M.H. "Instability of thixotropic fluids in pipe flow", MSc Thesis, University of Tehran (2015).

25. Whittington, R. and Ashton, E. "Instability in pipe flow", Nature, 162, pp. 997-998 (1948).

\section{Biographies}

Mohammad-Hosein Nahavandian is a PhD student of Mechanical Engineering at Amirkabir University of Technology, Tehran, Iran. He received MSc degree from University of Tehran and BSc degree from Iran University of Science and Technology, Tehran, Iran. His research interests include rheology, non-Newtonian fluid mechanics, instability, multiphase flows, CFD, Nano-fluids, and porous media.

Mohammad Pourjafar is a PhD student of Mechanical Engineering at University of Tehran. He received MSc degree from University of Tehran and BSc degree from University of Guilan, Tehran, Iran. His research interests are rheology, non-Newtonian fluid mechanics, poro-elastic instability, and peristaltic flows.

Kayvan Sadeghy is a Professor of Mechanical Engineering at University of Tehran. He received his $\mathrm{PhD}$ degree from University of Toronto, Toronto, Canada. He obtained his BSc and MSc degrees from University of Tehran, Tehran, Iran. He conducts experimental/numerical/analytical research in the fields of complex fluids, non-Newtonian fluid mechanics, and instability, among others. 